\title{
New $R B 1$ oncogenic mutations and intronic polymorphisms in Serbian retinoblastoma patients: genetic counseling implications
}

\author{
Milica Kontic · Iciar Palacios · Ángelo Gámez · Isabel Camino · \\ Zoran Latkovic · Dejan Rasic · Vera Krstic · Vera Bunjevacki · \\ Javier Alonso $\cdot$ Ángel Pestaña
}

Received: 19 April 2006/ Accepted: 5 July 2006/ Published online: 14 September 2006

(C) The Japan Society of Human Genetics and Springer-Verlag 2006

\begin{abstract}
The purpose of this work was to identify germ line $R B 1$ mutations in 16 Serbian retinoblastoma patients for genetic counselling. Mutation analysis was carried out by PCR directed sequencing of the 27 exons. Loss of heterozygosity for two $R B 1$ intragenic markers was also analyzed in 14 tumour samples. Five new $R B 1$ oncogenic mutations (g.2078 del C, g.77047_48 del GC, g.78117_8 del TT, g.160797 del T, and g.64439+2 T>C) and two recurrences (R445X and Q383X) have been found in this study. In addition, four intronic variants were observed germ line in some unilateral patients. Two of these variants (g.44668-15T/G, and g.166204$8 \mathrm{~T} / \mathrm{A}$ ) are discussed as potential oncogenic mutation candidates. The results show the relevance of studies aimed to investigate the role of intronic variants in exon splicing regulation. Such studies will help to disclose hidden retinoblastoma susceptibilities, important for accurate genetic counselling.
\end{abstract}

M. Kontic $\cdot$ V. Bunjevacki

School of Medicine,

Institute of Biology and Human Genetics, Belgrade,

Serbia and Montenegro

Z. Latkovic · D. Rasic · V. Krstic

Clinical Centre of Serbia, Institute of Ophthalmology,

Belgrade, Serbia and Montenegro

I. Palacios · Á. Gámez · I. Camino .

J. Alonso · Á. Pestaña

Institute of Biomedical Research "A. Sols",

CSIC-UAM, Madrid, Spain

\section{Á. Pestaña $(\bowtie)$}

Instituto Investigaciones Biomédicas,

C/ Arturo Duperier 4, 28029 Madrid, Spain

e-mail: apestana@iib.uam.es
Keywords $R B 1 \cdot$ Mutation screening $\cdot$ Intronic variants $\cdot$ Retinoblastoma $\cdot$ Serbian patients
Abbreviations
RBDMdb Retinoblastoma gene mutation database
WT Wild-type
$\mathrm{LOH} \quad$ Loss of heterozygosity

\section{Introduction}

Retinoblastoma (MIM\# 180200), is a rare embryonic neoplasm of retinal origin, with a relative incidence of $2.6 \%$ of all pediatric tumors (Narod et al. 1991), and an age standardized incidence of $4.1 \times 10^{6}$ in the $0-14$ years interval (Steliarova-Foucher et al. 2002). Most of the clinical phenotypes can be explained by the double mutational inactivation of $R B 1$, the retinoblastoma susceptibility gene (Cavenee et al. 1983), prototype of tumor suppressor genes that controls cell cycle progression (Kaelin 1999). According to the well established genetic model, in the hereditary form of the disease, a germ line mutation is transmitted as a high penetrance $(90 \%)$ autosomic dominant trait, resulting in a $45 \%$ risk in offspring of patients with hereditary retinoblastoma; the second inactivating mutation occurs in retinal cell precursors (Knudson 1971). Most of these patients have bilateral retinoblastoma and a mean age at diagnosis less than one year. In the nonhereditary form of the disease, both inactivating events occur during the somatic development of retinal cells and result in the relatively late onset of a single tumor 
in one eye (Draper et al. 1992). However, nearly $15 \%$ of the unilaterally affected patients have germ line $R B 1$ mutations, representing a $45 \%$ risk for their offspring, and these patients cannot be clinically distinguished from patients with true somatic unilateral retinoblastoma, who present a negligible risk for siblings and offspring (Murphree 1995; Harbour 1998). In addition, the presence of $R B 1$ germ line mutations confers an increased risk for development of second primary tumors, mainly osteosarcoma and other sarcoma, in the survivors of hereditary retinoblastoma, with a cumulative incidence of $22 \%$ at the age of 25 years (Moll et al. 2001). In addition, hereditary retinoblastoma survivors have a lifetime risk of developing common epithelial cancers (Fletcher et al. 2004).

In order to identify carriers and to provide accurate genetic counseling, we have undertaken a mutational study of $R B 1$ in Serbian retinoblastoma patients, referred to the Institute of Ophthalmology in Belgrade. Our results show the importance of mutational screening of unilateral retinoblastoma patients and the relevance of intronic variants as possible oncogenic mutations.

\section{Materials and methods}

The mutational studies were carried out in the peripheral blood DNA of 4 bilateral retinoblastoma patients and 12 unilateral patients, referred to the Institute of Ophthalmology, Belgrade. One of the bilateral patients has a family history. Written informed consent was obtained from the patients or their parents. Diagnosis of retinoblastoma was established by standard ophthalmologic and histological criteria. Paraffin embedded, histological representative, tumor samples from three bilateral and eleven unilateral patients were also available for study. In addition, a chorionic villous sample was provided for prenatal diagnosis. RNA for alternative splicing investigation was not available.

Whole blood from patients and relatives was collected in standard EDTA blood collection tubes and the DNA were extracted as described elsewhere (Alonso et al. 2001a, b). DNA was also extracted from paraffin embedded tumor sample using reagents and protocols provided by QIAGEN. Direct sequencing of PCR amplimers exons 2-27 was carried out as described (Alonso et al. 2001a, b). For exon 1 amplification, the following set of primers was used:

\section{RB 1F: 5'-TGTAAAACGACGGCCAGTTGTAAC GGGAGTCGGGA-3'}

RB 1R: 5'-CAGGAAACAGCTATGACCAACCC AGAATCCTGTCA-3'

Due to the high GC content, best results with exon 1 were obtained by using GC-RICH solution and Fast Star Taq DNA Polymerase, according to the protocol provided by the supplier (Roche Applied Sciences). All sequences were compared with the GenBank sequence (accession number: L11910). The mutations were sequenced at least once more from separate PCR reactions. The variation g.174361-10T/A was also tested by DraI restriction and electrophoresis of the PCR product as published (Alonso et al. 2001c).

Deletions involving intron 2 and/or intron 20 were analyzed in whole blood and tumor DNA samples, amplified with fluorescent-labeled primers specific for high polymorphic microsatellite markers Rbi2 and RB1.20, coupled to fragment analysis as described elsewhere (Alonso et al. 2001a, b).

When indicated, normal and mutated RB1 sequences were analyzed with the program Genscan available at the MIT web (http://www.genes.mit.edu/ GENSCAN.html), and the exon finder system available at the EMBO-EBI web (http://www.ebi.ac.uk/asdsrv/wb.cgi). Exonic splicing enhancers were analyzed with ESEfinder available at the CSH web (http:// www.ebi.ac.uk/asd-srv/wb.cgi).

\section{Results}

Seven small length mutations have been found in the mutational study of Serbian retinoblastoma patients (Table 1). The highly recurrent R445X mutation (24 hits in the Retinoblastoma gene mutation database, RBGMdb, by Valverde et al. 2005), has been observed germline in a bilateral patient and in one unilateral tumor. Another nonsense mutation, Q383X, found as second inactivating mutation in a bilateral tumor, has been previously reported twice (Richter et al. 2003). The four germ line frameshift deletions (g.2078delC, g.77047_48del GC, g.78117_8 del TT, g.160797_del C) found in patients SR15, SR21, SR23 and SR25 have not been reported before, according to data in RBGMdb (Valverde et al. 2005). The predicted outcome of these mutations will be inactive truncated $\mathrm{Rb}$ protein lacking one or both pocket domains. The somatic T/C transition (g.64439+2T $>$ C) affecting one invariant nucleotide at the splicing donor site of intron 10 (SR04T), will result in the deletion of exon 10 and a premature stop codon in exon 11.

Intragenic RB1 deletions affecting Rbi2 and/or RB1.20 markers were observed in six tumor samples 
Table 1 Oncogenic RB1 mutation in Serbian patients

\begin{tabular}{|c|c|c|c|c|c|c|c|}
\hline Patient & Laterality & Exon & Genomic description $^{\mathrm{a}}$ & $\begin{array}{l}\text { Predicted } \\
\text { consequences }\end{array}$ & Origin & Recurrencecs $^{c}$ & $\begin{array}{l}\mathrm{LOH} \text { in } \\
\text { tumor }\end{array}$ \\
\hline \multicolumn{8}{|c|}{ Peripheral blood DNA } \\
\hline SR25 & $\mathrm{U}$ & 1 & g.2078 del C & R7fsX55 & Germline & No & \\
\hline SR10 & B & 14 & g.76430 C>T & R445X & Germline & Yes (24) & \\
\hline SR21 & $\mathrm{BF}$ & 16 & g.77047_48del GC & S490fsX491 & Germline $^{\mathrm{d}}$ & No & \\
\hline SR15 & B & 17 & g.78117_8 del TT & V512fsX521 & Germline & No & \\
\hline SR23 & $\mathrm{B}$ & 21 & g.160797 del T & S725fsX743 & Germline & No & \\
\hline \multicolumn{8}{|c|}{ Tumor sample DNA } \\
\hline SR04T & $\mathrm{U}$ & 10 & g. $64439+2 \mathrm{~T}>\mathrm{C}$ & Exon 10 skipping & Somatic & No & Yes \\
\hline SR15T & $\mathrm{B}$ & 12 & g. $70261 \mathrm{C}>\mathrm{T}$ & Q383X & Somatic $^{\mathrm{e}}$ & Yes (2) & ND \\
\hline SR10T & B & 14 & g.76430 C>T & $\mathrm{R} 445 \mathrm{X}$ & Germline & Yes (24) & Yes \\
\hline SR02T & $\mathrm{U}$ & 14 & g.76430 C>T & $\mathrm{R} 445 \mathrm{X}$ & Somatic & Yes (24) & Yes \\
\hline SR23T & $\mathrm{B}$ & 21 & g.160797 del T & S725fsX743 & Germline & No & $\mathrm{RH}$ \\
\hline SR03T & $\mathrm{U}$ & & NF & & & & Yes \\
\hline SR22T & $\mathrm{U}$ & & NF & & & & Yes \\
\hline SR26T & $\mathrm{U}$ & & NF & & & & Yes \\
\hline SR07T & $\mathrm{U}$ & & NF & & & & $\mathrm{RH}$ \\
\hline SR29T & $\mathrm{U}$ & & NF & & & & NI \\
\hline
\end{tabular}

Abbreviations used are: $U$ unilateral sporadic, $B$ bilateral, $B F$ bilateral familiar, $N D$ not determined, $N F$ not found, $N I$ noninformative, $R H$ retention of heterozygosity

${ }^{a}$ Description follows the recommendations published by den Dunnen and Antonarakis 2000, using a genomic sequence (GenBank: L11910.1)

b Deduced changes at cDNA (NCBI: NM_000321.1) and protein level (NCBI: NP_000312.1)

c Number of occurrences (in brackets) in previously published $R B 1$ mutations (see text)

d Also found in unilaterally affected father. Not detected in chorionic villi of pregnant mother

e Second $R B 1$ inactivating mutation

(Table 1), leading to hemizygosity of the mutated allele (see an example in Fig. 1). In one tumor (SR15T), the complete inactivation of $R B 1$ function was achieved through a second small length mutation. In the tumor samples SR23T and SR07T no LOH of the polymorphic markers was observed. The second inactivating mutation in these cases could be the result of an intragenic deletion within exons 2 and 20. However, this possibility remains unexplored for insufficient genomic DNA sample. In five tumor samples from unilateral patients, shown in Table 1, PCR sequencing could not be performed. No reliable sequencing or fragment analysis could be performed in other four tumor samples due to extensive degradation.

Besides these canonical RB1 mutations, four intronic variants were observed in the Serbian study (see Table 2). A $\mathrm{T} / \mathrm{G}$ transversion at -15 in intron 4 (g.44668-15T $>\mathrm{G}$ ) has been observed germline in a unilaterally affected Serbian patient (SR08) and his unaffected father. This rare variant could not be detected in other 146 retinoblastoma patients from Spain, Colombia, Cuba and Serbia, in which exon 5 was sequenced. The variation g.166204-8T/A in intron 22 has been observed germline in a Serbian unilateral patient (SR016), with no other sequence alterations.
The intronic variants g.76869-14A/T and g.174361-10T/ A were observed in cases and controls with rather similar frequency and therefore represent true single nucleotide polymorphisms.

\section{Discussion}

The identification of germline mutations is important to detect affected or asymptomatic carriers within a pedigree, and also to provide prenatal or preimplantation genetic diagnosis. In this study, the oncogenic mutation detected in the patient with family history, was also found germline in the affected father, but not in the chorionic villi of the pregnant mother.

A true oncogenic mutation was found germline in one unilateral sporadic retinoblastoma patient (SR25) out of the twelve analyzed. Although the sample size is small, the observed frequency $(1 / 12=8.3 \%)$ is much lower than the $22.9 \%$ of germline mutations found by us in a larger unilateral retinoblastoma sample (Alonso et al. 2005). Candidates for oncogenic mutations can be found among the intronic variants which in several occasions have been found to alter RNA sequence motifs for exon splicing enhancers or silencers 

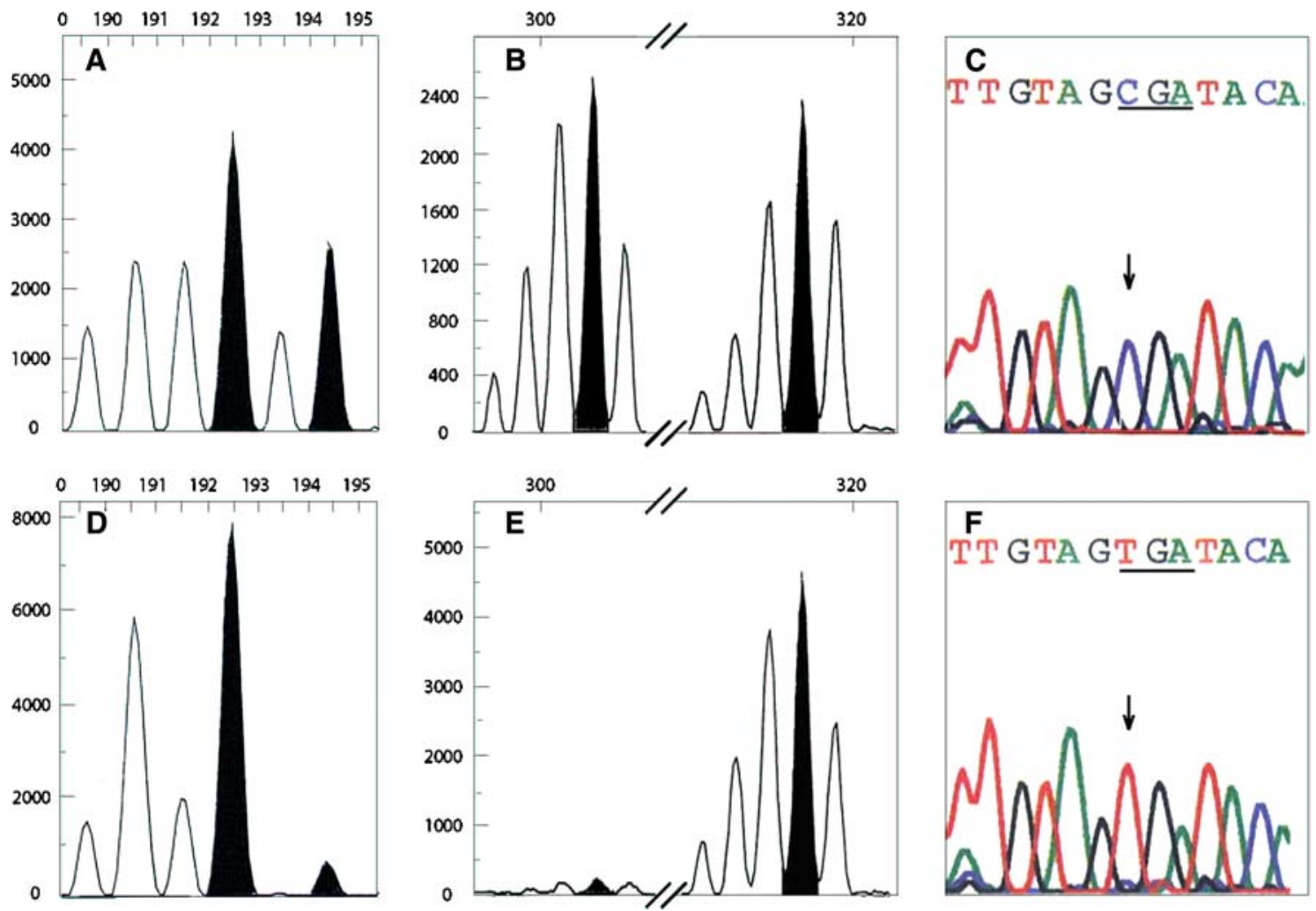

Fig. 1 Allelotype and sequence of peripheral blood and tumor DNA from patient SR02. Fragment analysis of markers Rbi2 a, d and RB1.20 b, e and exon 14 sequence $\mathbf{c}, \mathbf{f}$ in peripheral blood $\mathbf{a}-\mathbf{c}$ and tumor sample d-f DNA

Table 2 Intronic $R B 1$ polymorphisms found in Serbian patients

\begin{tabular}{|c|c|c|c|c|c|c|}
\hline \multirow[t]{2}{*}{ Intronic variants } & \multirow[t]{2}{*}{ Rare allele in Serbian patients ${ }^{\mathrm{a}}$} & \multicolumn{2}{|c|}{ Cases $^{\mathrm{b}}$} & \multicolumn{2}{|c|}{ Controls $^{\mathrm{c}}$} & \multirow[t]{2}{*}{$\mathrm{P}^{\mathrm{d}}$} \\
\hline & & $N$ & Rare allele frequency & $N$ & Rare allele frequency & \\
\hline g.44668-15T/G & $1 \mathrm{U}(\mathrm{SR} 08)$ & 146 & 0.007 & ND & ND & \\
\hline g.76869-14A/T & $4 \mathrm{U}(\mathrm{SR} 09,20,22,25)$ & 172 & 0.076 & 131 & 0.096 & 0.3834 \\
\hline g.166204-8T/A & $1 \mathrm{U}(\mathrm{SR} 16)$ & 184 & 0.016 & ND & ND & \\
\hline g.174361-10T/A & $4 \mathrm{U}(\mathrm{SR} 01,04,16,26), 1 \mathrm{~B}(\mathrm{SR} 23)$ & 135 & 0.241 & 58 & 0.207 & 0.5118 \\
\hline
\end{tabular}

$N$ number of cases or controls under study. $N D$ not determined

a Germline oncogenic mutations were found in patients SR25 and SR23. Abbreviations as in Table 1

b Variants found in patients from Spain, Colombia, Cuba and Serbia

c Healthy controls from Spain

${ }^{\mathrm{d}}$ Fisher exact test

(Cartegni et al. 2002). Our results (Table 2) have shown the presence of two intronic variants in Serbian patients, with potential oncogenic significance.

Several lines of evidence suggest the oncogenic character of the intron 4 variation, g.44668-15T $>$ G. Using the program Genscan for predicting the locations and exon-intron structures of genes in genomic DNA, it was observed that RB1 exon 5 is a suboptimal exon, which is skipped in silico in the $\mathrm{G}$ allele. With the help of the ESEfinder program for splicing enhancers' motif prediction, it was observed that the rare allele $G$ generates a functional SRp40 binding site (TCATAAG), located in g.44647-g.44653, with a score of 3.37 vs. 0.99 in the normal sequence (TCATAAT). This high affinity SRp40 motif in the intronic variant could activate alternative splicing mechanisms, as recently demonstrated for an intronic polymorphism in the KLF6 tumor suppressor gene (Narla et al. 2005). These in silico predictions have been confirmed in unpublished results with minigene constructions (A. Gámez et al. communication to the 19 European Association for Cancer Research meeting, Budapest, 
July 2006), and suggest the presumptive oncogenic character of the g.44668-15T $>\mathrm{G}$ variation.

In the rare variation g.166204-8T>A, the $\mathrm{T}>\mathrm{A}$ transversion (...tttttgttttt $>$ agctctagCCCCC...) reduces de consensus value $(\mathrm{CV}$, as defined by Shapiro and Senepathy 1987) from 80.6 to 69.5 , and creates a new splicing site 6 nucleotides upstream of the wild type acceptor site (tttttgttttagCTCTAGCCCCC...), with a better consensus value (77.6) than the mutant. The usage of this cryptic site is expected to result in a chain terminator two codons downstream. Although this prediction could not be tested at the present, for lack of RNA, functional studies with minigene constructions will be carried out in the future to test the hypothetic oncogenic character of this variation. The relevance of such studies for genetic counseling needs not be emphasized, as they could help to disclose hidden retinoblastoma susceptibilities.

Acknowledgments This work was supported by grants from the Comunidad de Madrid (GR/SAL/0855/2004), Ministerio de Educación y Ciencia (SAF2005-00946) and Instituto de Salud Carlos III (PI050128). IP held a postdoctoral contract from the Comunidad de Madrid; AG is a predoctoral fellow from the Ministerio de Educación y Ciencia; IC was contracted with a grant C03/10 from the Instituto de Salud Carlos III; MK held a short term fellowship (ICRETT 1029) from the UICC.

\section{References}

Alonso J, Mendiola M, García-Miguel P, Abelairas J, Pestaña A (2001a) A Microsatellite fluorescent methodology for linkage analysis studies in familial retinoblastoma and deletion detection at the $R B 1$ locus in retinoblastoma and osteosarcoma. Diagn Mol Pathol 10:9-14

Alonso J, Mendiola M, García-Miguel P, Abelairas J, Sarret E, Vendrell T, Navajas A, Pestaña A (2001b) Spectrum of germline $R B 1$ gene mutations in Spanish retinoblastoma patients: Phenotypic and molecular epidemiological implications. Hum Mutat 17:412-422

Alonso J, Mendiola M, Moreno C, López A, García-Miguel P, Abelairas J, Sarret E, Vendrell T, Navajas A, Pestaña A (2001c) Five new polymorphisms in $R B 1$ gene in retinoblastoma patients. Hum Mutat MPR \#237 Online

Alonso J, Frayle H, Menéndez I, López A, García-Miguel P, Abelairas J, Sarret E, Vendrell T, Navajas A, Artigas M, Torrenteras C, Pestaña A (2005) Identification of 26 new constitutional RB1 gene mutations in Spanish, Colombian and Cuban retinoblastoma patients. Hum Mutat 25(1): 99 Online

Cartegni L, Chew SL, Krainer AR (2002) Listening to silence and understanding nonsense exonic mutations that affect splicing. Nat Rev Genet 3:285-297
Cavenee WK, Dryja TP, Phillips RA, Benedict WF, Godbout R, Gallie BL, Murphree AL, Strong LC, White RL (1983) Expression of recessive alleles by chromosomal mechanism in retinoblastoma. Nature 305:779-784

Draper GJ, Sanders PA, Brownbill, Hawkins MM (1992) Patterns of risk of hereditary retinoblastoma and applications to genetic counseling. Br J Cancer 66:211-219

Fletcher O, Easton D, Anderson K, Gilham C, Jay M, Peto J (2004) Lifetime risks of common cancers among retinoblastoma survivors. J Natl Cancer Inst 96:357-363

Gallie BL, Campbell CH, Devlin H, Duckett A, Squire JA (1999) Developmental basis of retinal-specific induction of cancer by RB mutation. Cancer Res 59:1731s-1735s

Harbour JW (1998) Overview of RB gene mutations in patients with retinoblastoma. Ophthalmology 105:1442-1447

Kaelin WG (1999) Functions of the retinoblastoma protein. Bioessays 21:950-958

Knudson AG (1971) Mutation and cancer: statistical study of retinoblastoma. Proc Natl Acad Sci USA 68:820-823

Moll AC, Imhof SM, Schouten-Van Meeteren AYN, Kuik DJ, Hofman P, Boers M (2001) Second primary tumors in hereditary retinoblastoma: a register-based study, 19451997. Ophthalmology 108:1109-1114

Murphree AL (1995) Molecular genetics of retinoblastoma. Ophthalmol Clin North Am 8:155-166

Narla G, DiFeo A, Reeves HL, Schaid DJ, Hirshfeld J, Hod E, Katz A, Isaacs WB, Hebbring S, Komiya A, McDonnell SK, Wiley KE, Jacobsen S, Isaacs SD, Walsh PC, Zheng S, Chang B, Friedrichsen DM, Stanford JL,Ostrander EA, Chinnaiyan AM, Rubin MA, Xu J, Thibodeau SN, Friedman SL, Martignetti JA (2005) A germline DNA polymorphism enhances alternative splicing of the KLF6 tumor suppressor gene and is associated with increased prostate cancer risk. Cancer Res 65:1213-1222

Narod SA, Stiller C, Lenoir GM (1991) An estimate of the heritable fraction of childhood cancer. Br J Cancer 63:993999

Shapiro MR, Senepathy P (1987) RNA splice junctions of different classes of eukaryotes: sequence statistics and functional implications in gene expression. Nucleic Acid Res 15:7155-7174

Steliarova-Foucher E, Berrino F, Coebergh JW, Kaatsch P, Lacour B, Michaelis J, Mitton N, Stiller CA, Parkin DM (2002) ACCISpass 1.01, software for analysis and presentation of data on incidence and survival of children and adolescents in Europe. Lyon: European Network of Cancer Registries

Richter S, Vandezande K, Chen N, Zhang K, Sutherland J, Anderson J, Han L, Panton R, Branco P, Gallie B (2003) Sensitive and efficient detection of RB1 gene mutations enhances care for families with retinoblastoma. Am J Hum Genet 72(2):253-69

Valverde JR, Alonso J, Palacios I, Pestaña A (2005) RB1 gene mutation up-date. A meta-analysis based on 932 reported mutations available in a searchable database. (RBGMdb; http://www.es.embnet.org/Services/MolBio/rbgmdb) BMC Genetics 6:53 